\title{
A Practical Decision Tool to Evaluate and Rank Potential Solutions for Expected Downhole Drilling Problems During the Well-planning Phase
}

\author{
Asad Elmgerbi ${ }^{1}$ (D) Borna Les ${ }^{1} \cdot$ Rahman Ashena $^{2} \cdot$ Timothy Atkin $^{3}$
}

Received: 19 January 2021 / Accepted: 16 December 2021/Published online: 16 February 2022

(C) The Author(s) 2022

\begin{abstract}
Currently, the main objective of oil operating companies is to increase drilling efficiency and minimize drilling costs; this can be achieved in several ways. One of them is the curtailment of downhole events associated with trouble time by obtaining the key factors that drive the best proactive solutions in terms of cost and effectiveness. From this perspective, the core focus of this paper is to present a detailed description to develop an integrated decision tool that will assist well planner engineers in evaluating multiple solutions considered to address a downhole problem anticipated during the well construction process. The tool presented here provides the means of assessing a particular solution's cost and anticipated effectiveness by generating an evaluation matrix based on two intercorrelated decisiondriving indices, technical and monetary. To theoretically validate the concept, an artificial case study was created to find the best solution in mitigating a bit balling problem. The results of the case study reveal that: the concept is indeed theoretically applicable. However, a real life case study must be performed to prove the concept practically.
\end{abstract}

Keywords Decision Tools · Downhole Drilling Problems · Decision Quality · Drilling Trouble Time

Asad Elmgerbi

asad.elmgerbi@unileoben.ac.at

1 Montanuniversität, Leoben, Austria

2 Asia Pacific University of Technology \& Innovation (APU), Kuala Lumpur, Malaysia

3 AGR Software, Oslo, Norway

\section{Introduction}

To meet the global demand for a steady supply of energy at affordable prices, the industry is now faced with the choice of developing technologies that can withstand a new era of drilling challenges. As the drilling costs significantly contribute to the cost per barrel, the industry is pressured into finding ways to lower the cost per foot drilled. Furthermore, hydrocarbon reservoirs are depleting faster than ever before, and the remaining plays are becoming technically more and more difficult to access. Well, construction becomes more and more complex as more extreme profiles like Deepwater subsalt plays, extended reach, high-pressure high temperature, and remote arctic environments are being explored. To better plan for potential overruns, operators sometimes earmark up to $25 \%$ of the Authorization for Expenditures (AFE) to cover unexpected costs, which can significantly impact drilling budgets [1]. In understanding potential problems, the anticipation and planning for a solution becomes essential to control overall well cost and succeed in reaching the intended target [2]. The possibility of the aforementioned threats may be slight in a particular well design, but there are configurations where potential drilling problems can be easily foreseen during the planning stage due to for instance, geological context [3]. To compete in narrow economic windows, operators should aim to mitigate difficult drilling conditions and improve the wells economics by employing the most effective solutions for a particular cause and avoid reaching the contingency fund within a well's AFE. Due to the ubiquitous oil price fluctuations and complicated engineering applications, the selection of downhole decision tools and tuning drilling decisions should be harmonized to diminish downhole problems and failures while minimizing non-productive time (NPT). 
Proactive solutions for combating downhole drilling problems are considered more favorable as they reduce the likelihood of occurrence rather than minimize the impact of an unanticipated problem [4, 5]. However, when multiple proactive solutions are available for a given downhole drilling problem, the considered solutions should be riskweighted for determining the most economical option [6]. Therefore, Over the last decade, various methodologies emerged as decision tools helping to obtain the optimum proactive solution for encountering operational problems. In the next section, the most relevant tools are discussed.

Knowledge-intensive Case-Based Reasoning (KiCBR) is a problem-solving and decision-making approach. The encountered problem is solved by finding a set of similar previously solved problems, called cases, and reuse them to solve the newly faced problem [7]. The KiCBR was recently applied in a case study to determine the root cause of poor hole cleaning episodes [2]. However, the proposed methodology acts as an advisor in retrieving similar cases and listing the results to enable root cause prediction. Incorporating an extensive database to successfully establish similarities between matched well sections, as well as a lack of risk and cost assessment on a particular decision, is considered as the main drawback.

The first application of machine learning in cotext of real time drilling decision support was presented in 2013. The proposed approach utilized machine learning to construct a KiCBR platform for comparing a real-time situation with historical cases where problems occurred to differentiate between best drilling practices and building a fast, practical, real-time solutions. Although the approach can provide fast solutions, it fails in evaluating the solutions in terms of expected cost and effectiveness [8].

Other concepts used in support of drilling decisions are so-called Influence diagrams or Bayesian networks. Over time, the usage of Bayesian Networks prevailed over classic decision trees, providing a level of insight and transparency in more complex decisions [9]. The use of influence diagrams based on the mathematical apparatus of Bayesian networks (BN) allows a combination of graphical representations with its probabilistic nature and evaluation of a decision utility. Unlike KiCBR methods, the apparatus of $\mathrm{BN}$ provides a theoretical approach and a mathematically proven method that is sufficiently accurate in extremely complex situations and conditions with predominant uncertainty [10].

In 2017, a method to identify, assess, prioritize, and manage drilling risks based on engineering judgment and expertise was presented. It implemented the analytical hierarchy process to rank previously filtered hazards, termed Risk Influencing Factors, consequently adducing total risk exposure for a particular problem without any costeffective criterion [11].
Another widely used methodology for drilling decisions is quantitative risk assessment. Although this method has shown its applicability within risk assessment, it cannot be considered idealistic due to the following: A lack of systematic approach to rank the possible solutions in terms of effectiveness and efficiency, unconsidered uncertainty associated with cost estimation for implementing a particular solution for a specific downhole problem, and a methodology to estimate the impact of potential solutions during the well construction planning phase [12].

An approach based on game theory was recently introduced as a stochastic decision analysis model to estimate the risk of downhole tool failures. The presented work assesses the tool reliability and replacement risk and simulates the possible outcomes with different drilling plans and tool maintenance schedules. The stochastic approach was shown helpful in identifying high-risk tools and their components by generating Pay-off tables for a planned drilling run based on the replacement cost, the probability of failure $(\mathrm{PoF})$, and the extra incurred cost due to occurred failure. It can be stated that the authors credibly argued a possible usage of game theory to minimize downhole tool failures. However, a limited spectrum of applications and low relation arise when considering the overall drilling costs, which is the main disadvantage of the proposed concept [13].

With the encompassing digitalization of the upstream sector, many machine learning tools for predictive methods to identify possible NPT-related events during well construction are developed. However, these software tools likely cannot fully replace manual analysis by subject matter experts (SME) in real-time; instead, they augment the SME's ability to identify the correlations in a large volume of data. As these systems are being developed, an SME can benefit from a centralized database that contains the ever-increasing amount of digital data collected during well construction. Although the decision-makers are provided with data-supported recommendations at crucial junctures, these recommendations typically involve costly rig time with ongoing drilling activity. The trade-off between added rig time and benefits gained from the "machine" recommendation is difficult to quantify. Undoubtedly, operators will benefit from addressing downhole problems yet due to robust pre-planning. The emphasis should be put on avoiding or preventing the problem rather than early detection. One cannot disregard that such tools can augment the well construction process in real-time by supporting the recommendation. However, as a more thorough assessment is conducted during the planning phase, the less inherent uncertainty there will be, which will, in turn, reduce the likelihood of crucial junctures, thus causing less rig downtime [9, 14, 15 and 16]. 
This papers aim is to introduce a new decision tool to enhance the decision-making process and deliver more positive results during the well planning phase; by employing semi-quantitative and stochastic methods, the evaluation matrix is developed comprising two indices to provide a quantitative basis for selecting the most foreseen optimal drilling solution from technical and economic perspectives.

\section{Background}

Overlooking particular risks or failing to address all the possible outcomes during the well planning process can bring significant errors in the cost and time estimation of the performed drilling operation. Eventually, this may impact the overall project economics to a considerable extent. The uncertainty associated with cost estimation for a particular solution during the planning phase is very high. The solution impact can be measured only once the solution has practically been implemented. To determine the optimum proactive solution in terms of costs and effectiveness, the presented integrated decision-making tool relies on using two indices, a Technical indices (TI) and a Monetary indices (MI), to rank the possible proactive solutions for a given downhole problem.

Technical Indices predict how well the nominated proactive solution combats a particular downhole problem. It reflects only the technical aspect of the solution. Although different tools may be adapted to estimate the technical indices, due to its simplicity and aptness for making complex decisions Weighted Matrix Method (WMM) was selected to be used in this study. In contrast, the Monetary Indices assesses the anticipated cost and benefit of the studied solutions. Ultimately, the two generated indices can be plotted against each other in a $2 \mathrm{D}$ evaluation matrix, which provides the final score one could use to screen multiple solutions in terms of cost and

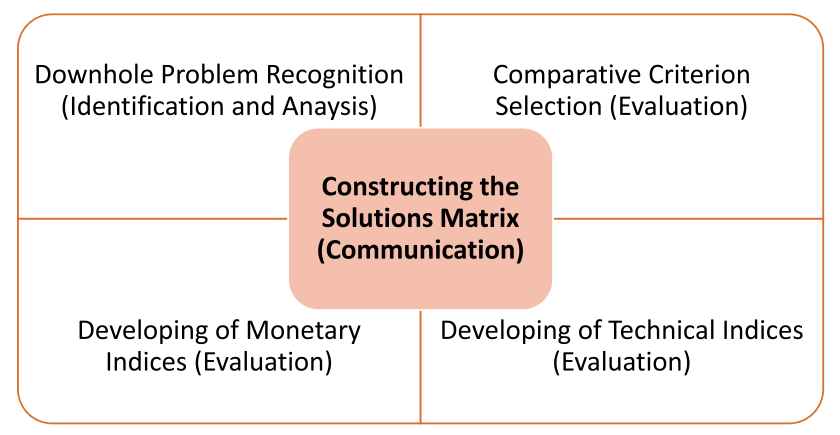

Fig. 1 Main processes followed to develop an integrated decisionmaking tool effectiveness. Figure 1 depicts the illustration workflow of the proposed methodology.

\section{Downhole Problem Recognition and Solutions Identification}

The most commonly used approach for identifying the likely downhole problems and their root causes is by analyzing historical data ( offset well trouble time). The trouble time analysis process helps outline critical downhole problems that could cause delays in planned drilling operations. Once the problems are defined, the involved person can move to the second step, in which the search for idealistic proactive solutions is started. Based on the nature of the problems and the causes, several potential solutions might be available. However, the question still open here is which solution will be more effective in terms of cost and competence.

\section{Comparative Criteria Selection}

The suggested criteria used in the rating of the TI and MI are listed below. Bear in mind that the authors propose the indicated list of criteria, but is not limited to; thus, the list could be extended further based on the expected downhole problems and the possible solutions. However, only a few criteria have been introduced here, selected based on the hypothetical case study, which will be explained later.

Criteria Proposed to Develop Technical Indice

I. Enhanced Rate of Penetration (EROP) represents the applied solution's effect on ROP enhancement. Due to that, some applied downhole solutions might have either a negative or positive influence on ROP.

II. Method Reliability (MR) represents the expected success rate while tackling the anticipated downhole problem. The solution is considered $100 \%$ effective if it is expected to reduce the NPT associated with the drilling problem to a minimum.

III. Downhole Impact (DI) indicates a level of impact delivered to the downhole performance in the overall drilling process. The values assigned to this criterion can be obtained only based on practical experience.

IV. Improved Wellbore Stability (IWS) shows the impact each solution will have on the wellbore stability. Precisely, studied solutions might have either positive or negative impacts on wellbore stability.

V. Solution Complexity (SC) accounts for the effort required to implement a particular solution. Higher the effort, higher the personnel's pressure, which may negatively affect flat time operations and increase overall drilling cost. Compared with other criteria in use, a simple ranking was used to assess the value 
entered into the WMM. SC is considered to harm the considered solution as opposed to other criteria, meaning the lower the weighted score, the better.

\section{Criteria Proposed to Develop Monetary Indice}

I. NPT Reduction Savings (NPTRS) This indicates the expected reduction in NPT that could be achieved by implementing the solution. It is calculated by multiplying the expected reduction in NPT by the estimated drilling rig hourly rate.

II. Extra Incurred Expense (EIE) refers to the direct expenditure required to implement the selected solution. By expressing incurred expense in unit cost, the total expense can easily be calculated by multiplying the unit price with the quantity required.

III. Enhanced Penetration Savings (EPS) indicates additional savings obtained through an increase in average ROP, which will eventually reduce total Invisible Lost Time (ILT). It can be estimated by multiplying predicted saved hours by the estimated drilling rig hourly rate.

IV. Implementation Estimated Time (IET) indirectly costs incurred due to time consumed while implementing a specific solution. It can be computed by using the estimated drilling rig hourly rate.

\section{Development of the Technical Indice (TI)}

As mentioned earlier, the Weighted Matrix (WM) is a simple semi-quantitative method used to manage or make a set of complex decisions. A filtering criterion can be established with numerous alternatives to rank all the accounted alternatives to the primary reference.

Using the known WM procedures for developing the TI is not possible. Consequently, additional steps are required to make the implementation of this tool applicable. The modified steps are outlined below:

1. Construct a list of all possible solutions for a particular downhole risk or hazard

2. Define a set of criteria using the appropriate benchmark that will allow comparison between different solutions

3. Evaluate each of the considered solutions separately for every introduced criterion

4. Assess the impact of each considered solution against a particular criterion, i.e., compare the impact of the individual solution score to benchmark within a set of listed solutions

5. To obtain a rating scale, assign a normalized weighted factor based on each criterion's importance and influence on bottom-line decision
6. Calculate weighted scores by multiplying the score of each solution by the weighted factor assigned

7. Provide a final comparison score for each alternative by adding all the calculated weighted scores for a particular drilling solution.

8. By using a Weighted Matrix with a pre-defined set of comparative criteria, TI can be computed.

\section{Development of Monetary Indices (MI)}

Before commencing the process of developing MI, the continuous probability distribution of each involved variable has to be defined. Two stochastic functions can describe the continuous probability distribution of any variable. The first one is the Probability Density Function (PDF), showing variables of interest with its frequencies. PDF is used to specify the probability of the random variable falling within a particular range of values instead of taking on any value. This probability is given by the integral of this variable's PDF over that range, viz, it is given by the area under the density function and between the lowest and greatest values of the range. Another function to describe the distribution of random variables is the Cumulative Density Function (CDF), denoting the probability the variate will take the value $x$. A scalar continuous distribution is given by the area under the probability density function from minus infinity to $\mathrm{x}$ or simply by calculating the area leftward from the variable of interest under the curve in the probability density function diagram. In the proposed tool, triangular distribution, often called "lack of knowledge distribution," was employed to reasonably represent the probability density function. Defined as a continuous probability distribution with a lower limit $\mathrm{a}$, upper limit $\mathrm{b}$, and mode $\mathrm{c}$, where $\mathrm{a}<\mathrm{b}$ and $\mathrm{a} \leq \mathrm{c} \leq \mathrm{b}$, it is based on a knowledge of the minimum and maximum and an "inspired guess." Thus, the assumption is made that the project planning team guessed for at least three values to define the triangular distribution [17].

In order to compute MI, two different target groups are set based on the summation of comparative criteria. The first group consists of criteria that represent the potential to reduce the associated costs. Since they are considered to add value to the selected method, they will be referred to as Positive Indicators (PI), while their summation can be considered as Foreseen Savings Potential. These include NPTRS and EPS (Fig. 2, blue-filled squares). In contrast, the second group comprises criteria that cause additional costs are referred to as Negative Indicators (NI). These include EIE and IET (Fig. 2, white squares); collectively, they will be used to express the Expected Expenses. The Summation costs are calculated by multiplying a unit cost and the effect each criterion has on the solution cost in 


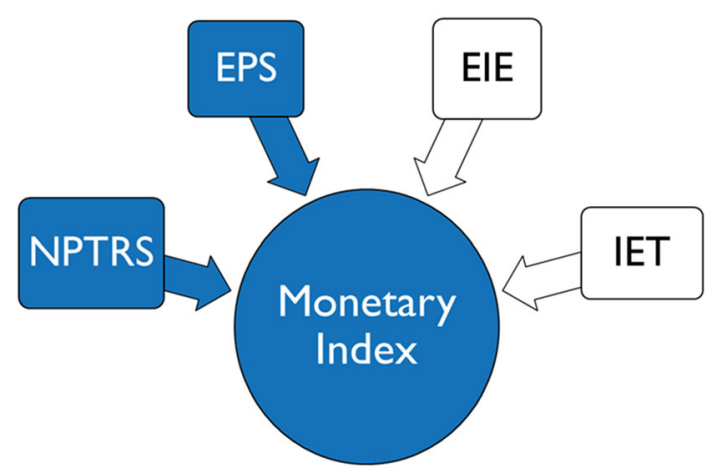

Fig. 2 Comparative criteria included in economic impact (Monetary Indice) assessment

terms of hours reduced/incurred. Figure 2 schematically summarizes criteria taken into developing MI. Once the target groups are set as Monte Carlo simulation (MCS) output, one can calculate the probability distribution for Foreseen Savings Potential and Expected Expenses. CDF is defined for Expected Expenses by simple multiplication of discrete probability values, ranging from $0-1$, and inferred from MCS to assess the MI value.

Similarly, however in the opposite range, i.e., 1-0, Reverse Density Function (RDF) is defined for the Foreseen Savings Potential. Such arrangement allows inferring the probability at which both functions coincide, thus representing the probability that the required cost for a particular solution will equal the savings cost. An example can be seen in Fig. 3.

It is essential to mention that there might be situations where the concurring probability may not be estimated from MCS output. Here, the following may be the causes:

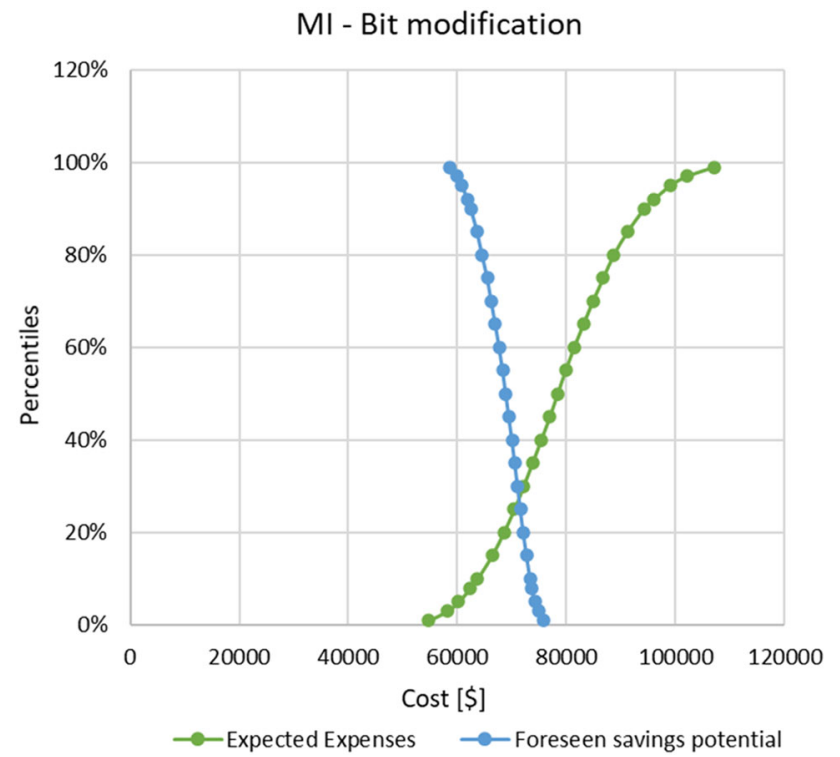

Fig. 3 Graphical method of assessing Monetary Indice (MI); statistical functions " plot to estimate the concurring probability
1. A relatively small economic impact, meaning the implemented solution will certainly be paid off (Fig. 4).

2. Expected Expenses increase to an extent where no Foreseen Savings will equal incur costs regardless of probability (Fig. 5).

The main steps to develop the MI are summarized in Fig. 6.

\section{Assessing the Decision Quality (Solution Matrix)}

By plotting rated indices against each other, a 2D evaluation matrix for the studied downhole problem. As illustrated in Fig. 7, users can distinguish between four areas, where each is labeled in terms of Technical Impact and Pay-off Probability.

The upper rightmost quadrant is of the highest interest, showing both the highest performance and pay-off probability. Conversely, with properly evaluated solutions, the drilling team should avoid any solution(s) positioned in the lower leftmost quadrant, representing alternatives with poor performance and high economic uncertainty. Using such a visualization technique simplifies the overall evaluation of the different solutions. It must be stated that having a highly rated Indice does not necessarily mean the proposed solution is adequate. For instance, if the solution has a very high Technical Indice but a low Monetary Indice, that indicates a costly solution, regardless of its technical impact, and vice versa. On the contrary, having a highly rated solution with both indices will ensure its

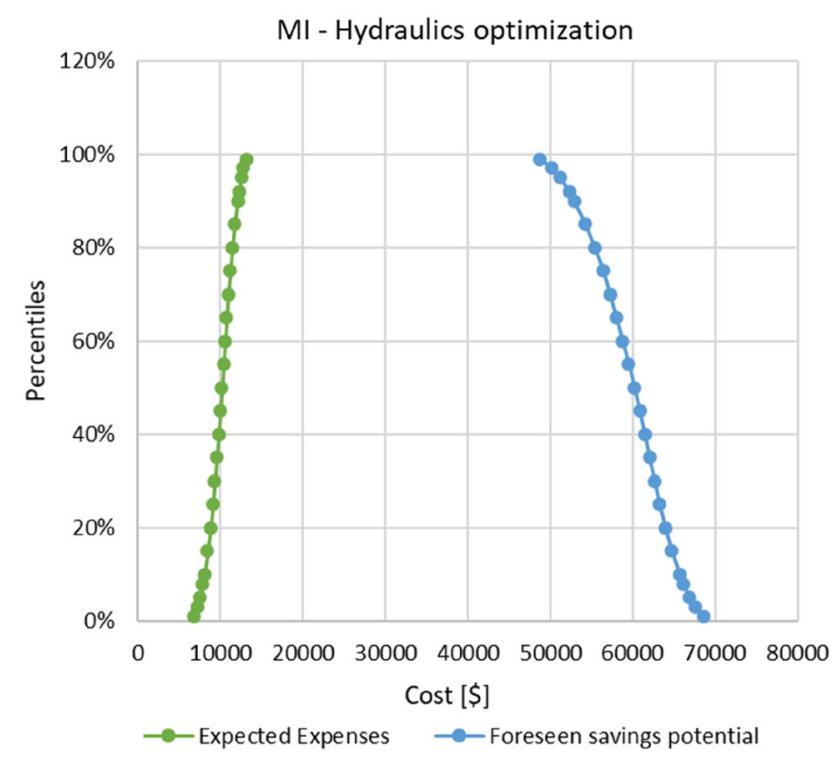

Fig. 4 Plot with no concurring probability to demonstrate the small economic impact (cause 1) 


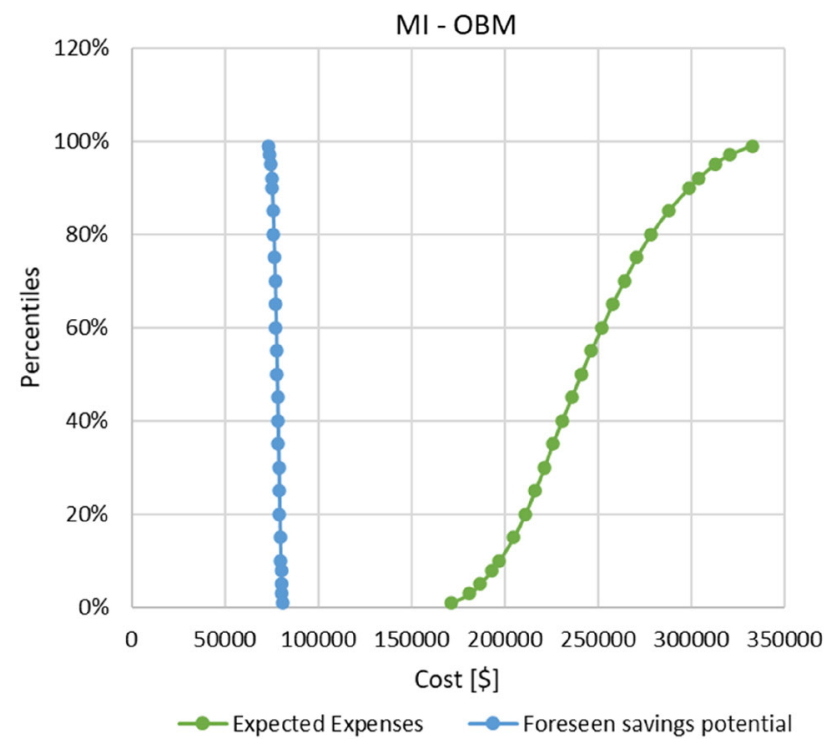

Fig. 5 Plot with no concurring probability to demonstrate the extensive economic impact (cause 2)

performance is most likely to be technically successful and economically feasible.

However, the planning team may come into a situation where proposed solutions will be clustered in the rightuppermost section. As displayed in Fig. 8, the most appropriate should be found among multiple alternatives. As shown in Fig. 8, solution A is the most cost-effective solution for this particular situation, although other solutions show outstanding performance. Due to the highest value of TI and MI, solution A should perform best not just from the technical point of view, i.e., mitigating anticipated problems, but also with the highest probability that the total incurred cost will equal foreseen savings. To conclude, evaluating possible drilling solutions and understanding compromise between the options themselves enables the team to prearrange the most appropriate and efficient technological solution for a particular cause.

\section{Result and Discussion}

A made-up case study related to bit balling was performed using a synthetic dataset to identify the shortcomings and added values of the developed tool. All the processes were conducted using open-source Model-Risk Software [18].

\section{Assumptions}

To develop a solutions matrix for the bit balling issue, several assumptions were made:

- Rig Hourly Rate (RHR) of 1250 \$ used to calculate relevant savings/costs while rating MI

- recorded total NPT of $130 \mathrm{~h}$ in which bit balling accounts for $60 \mathrm{~h}(46 \%)$. This assumption was necessary to evaluate different solutions in terms of NPT reduction and attributed savings

- assumed invisible lost time (ILT) of $87 \mathrm{~h}$ in which ROP accounts for $15 \mathrm{~h}(17 \%)$ was used while rating MI to assess the savings made through reduced penetration time or simply, EPS
Fig. 6 Steps developed to compute Monetary Indice

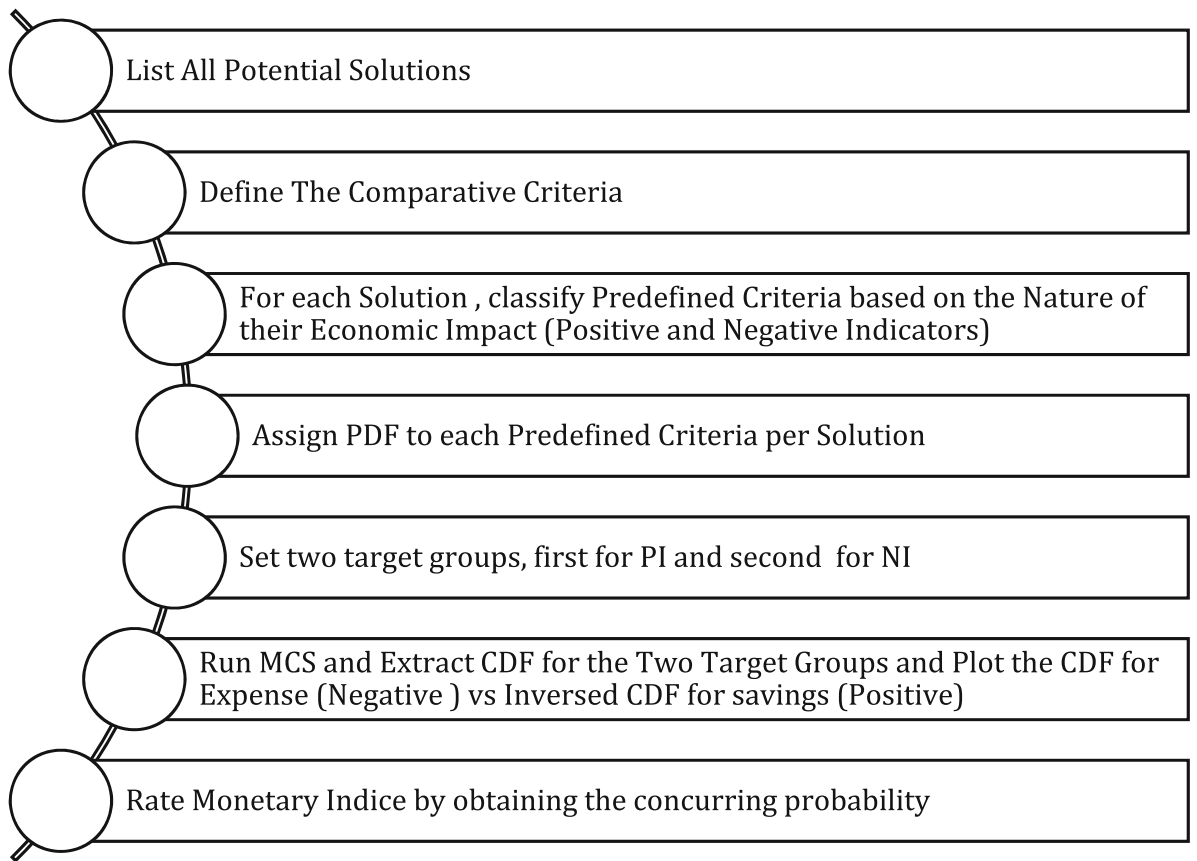


Fig. 7 Schematic Display of the Integrated Decision-Making Tool Solution Matrix
Fig. 8 Integrated decisionmaking tool solution matrix illustrative example
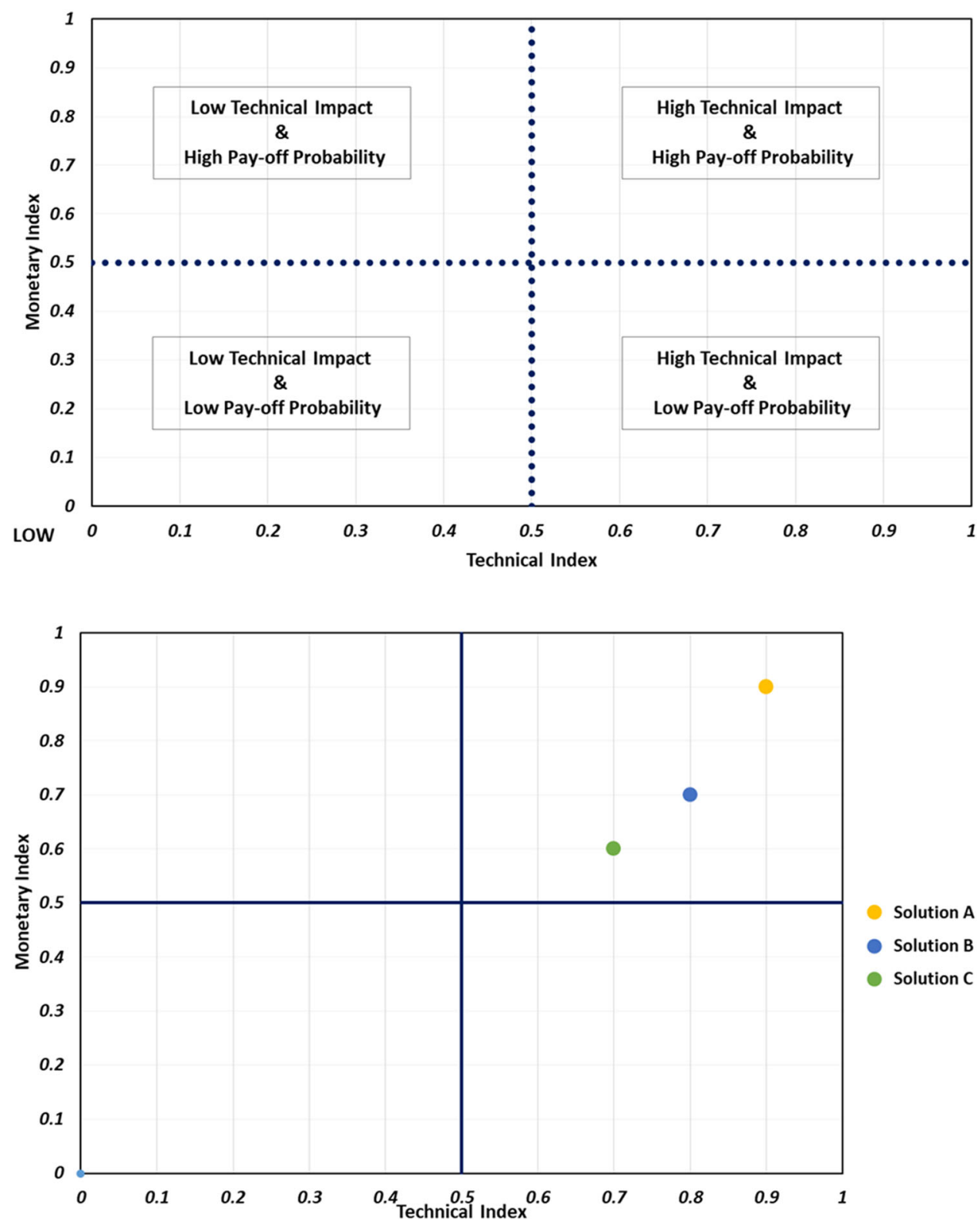

- total drilling fluids Volume of $2000 \mathrm{bbl}$, used to estimate implementation expenses (EIE) related to drilling mud design

- the length over which bit balling can be encountered was set across the interval of $1600 \mathrm{~m}$,

- a varying number of bits to be modified, thus affecting PDF assigned to EIE

- Min, Mean, and Max estimate for triangular distribution were obtained conferring people from the industry to obtain reasonable output throughout the Integrated Decision-Making Tool

- P50 used as a statistical indicator to evaluate TI

\section{Proposed Solutions}

Several proactive solutions were introduced in the last decade to mitigate the bit billing-related problems. However, only the most practical ones will be evaluated here; these are [19 and 20]:

- Bit Modifications (BM) represents upgrading from conventional PDC bits design to bits with a more aggressive penetration profile and geometry suitable to sticky formations. Features such as large open face volume and junk slot area, optimized nozzle placement, adequate hydraulic horsepower will minimize the bit balling effect. In addition, sharp polished cutters, aggressive rake angles, and edge geometry may 
significantly enhance chip management. However, this requires modifications that add substantial cost to bit procurement.

- Hydraulics Optimization (HO) This is referred to as an increase of Hydraulic Power delivered to the bit. Through the optimization, induced zones of high vortices will quickly and effectively remove cuttings beneath the bit, preventing bit balling.

- Improved Water-Based-Mud (IWBM) accounts for design improvement of deployed mud. The aim is to mitigate problems induced by sensitive shales' behavior such as sticking, agglomeration, and accretion through optimization of the mud chemistry.

- Oil Based Mud (OBM) This solution presenting the change of the entire drilling fluid from water-based to oil-based.

- Anti-balling Coating $(A b C)$ indicates special coatings used to prevent adhesion of shales on the bit surface, such as Electro-Osmosis or Enforced Fluoropolymer coating.

\section{Development of Technical Indices}

Five different criteria were selected to evaluate the mentioned solutions. Weighted Scores were calculated accordingly to Eqs. (1) and (2) (Refer to the Appendix). The TI value was assessed by summing the Weighted Scores as shown in the Sum (TI) column (See Fig. 9). Each of the scores was rated based on the P50 output from the MCS, performed using the ModelRisk Software. It is important to emphasize that, to make the assigned factors suitable for TI rating, Min-Max Feature Scaling was used to bring all values into the normalized range from 0 to 1 . In contrast, the scale used in ranking the solutions was arbitrarily set in the range from 1 to 10 . A schematic display was added to the leftmost column to ease the visualization within the rows presenting different solutions. Considering a defined set of Comparative Criteria and WF assigned, the highest value of the Technical Indice being 0.83. indicate OBM to be the most appropriate drilling solution to tackle the bit balling problem. Conversely, Bit Modification has the smallest TI; thus, it is considered the least effective in mitigating the same problem.

\section{Development of Monetary Indices}

According to the criteria outlined in the Comparative Criteria Selection, group constituents used in MI rating were set as given by Fig. 2. Each indicator was estimated over the reasonable range of costs incurred/deducted to/ from the overall well construction process. By summing the criteria over each group, two target groups, Expected Expenses, and Foreseen Savings Potential, were defined for simulation. The simulation was run for 10,000 trials to yield accurate PDF and CDF for both pre-defined target groups. This allows assessment of the percentage of Expected Expenditures and Foreseen Savings in its frequency distribution equal to or lower than its value. The visual representation of simulation results is given through Pareto Plots (Fig. 10).

The Foreseen Savings Potential had to be inverted to find the concurring probability, thus allowing one to estimate the point at which the value of a particular solution's total incurred cost will be equal to the value of the expected savings (a bit modification Monetary Indice). Monetary Indice can be easily found through the iterative procedure using MCS output; however, a graphical solution was proposed to ease the reader's comprehension.

As shown in Fig. 11., having both CDF and RDF plotted at the same graph, MI was assessed from a crossing of both

\begin{tabular}{|c|c|c|c|c|c|c|c|c|c|}
\hline \multirow{14}{*}{ 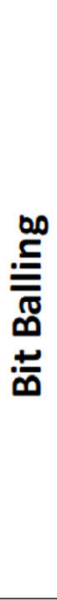 } & \multirow{4}{*}{$\begin{array}{c}\text { Considered } \\
\text { Solutions }\end{array}$} & $\begin{array}{c}\text { Comparative } \\
\text { Criterion }\end{array}$ & EROP & MR & DI & IWS & SC & Sum (TI) & $\begin{array}{c}\text { Value } \\
\text { Distribution }\end{array}$ \\
\hline & & Assigned WF & 4 & 6 & 8 & 5 & 2 & 25 & \\
\hline & & Normalized WF & 0.16 & 0.24 & 0.32 & 0.20 & 0.08 & 1.0 & \\
\hline & & Reference value & $\max$ & $\max$ & $\max$ & $\max$ & $\min$ & I & 1 \\
\hline & \multirow{2}{*}{ Bit Modifications } & P50 Value & 2.11 & 8.60 & 0.70 & 4.76 & 2.00 & \multirow{2}{*}{0.43} & \\
\hline & & WS & 0.04 & 0.21 & 0.02 & 0.11 & 0.04 & & \\
\hline & \multirow{2}{*}{$\begin{array}{c}\text { Hydraulics } \\
\text { Optimization }\end{array}$} & P50 Value & 1.19 & 7.73 & 4.80 & 5.93 & 1.00 & \multirow{2}{*}{0.60} & \\
\hline & & WS & 0.02 & 0.19 & 0.16 & 0.14 & 0.08 & & \\
\hline & \multirow{2}{*}{$\begin{array}{l}\text { Improved Water- } \\
\text { Based-Mud }\end{array}$} & P50 Value & 1.63 & 9.05 & 8.16 & 8.22 & 5.00 & \multirow{2}{*}{0.74} & \\
\hline & & WS & 0.03 & 0.23 & 0.28 & 0.19 & 0.02 & & \\
\hline & \multirow{2}{*}{ Oil Based Mud } & P50 Value & 3.45 & 9.64 & 9.38 & 8.50 & 8.00 & \multirow{2}{*}{0.83} & \\
\hline & & WS & 0.06 & 0.24 & 0.32 & 0.20 & 0.01 & & \\
\hline & \multirow{2}{*}{ Antiballing Coating } & P50 Value & 8.77 & 9.33 & 7.80 & 5.13 & 2.50 & \multirow{2}{*}{0.81} & \\
\hline & & WS & 0.16 & 0.23 & 0.27 & 0.12 & 0.03 & & \\
\hline
\end{tabular}

Fig. 9 A Bit Balling Weighted Matrix and TI values for considered solutions 


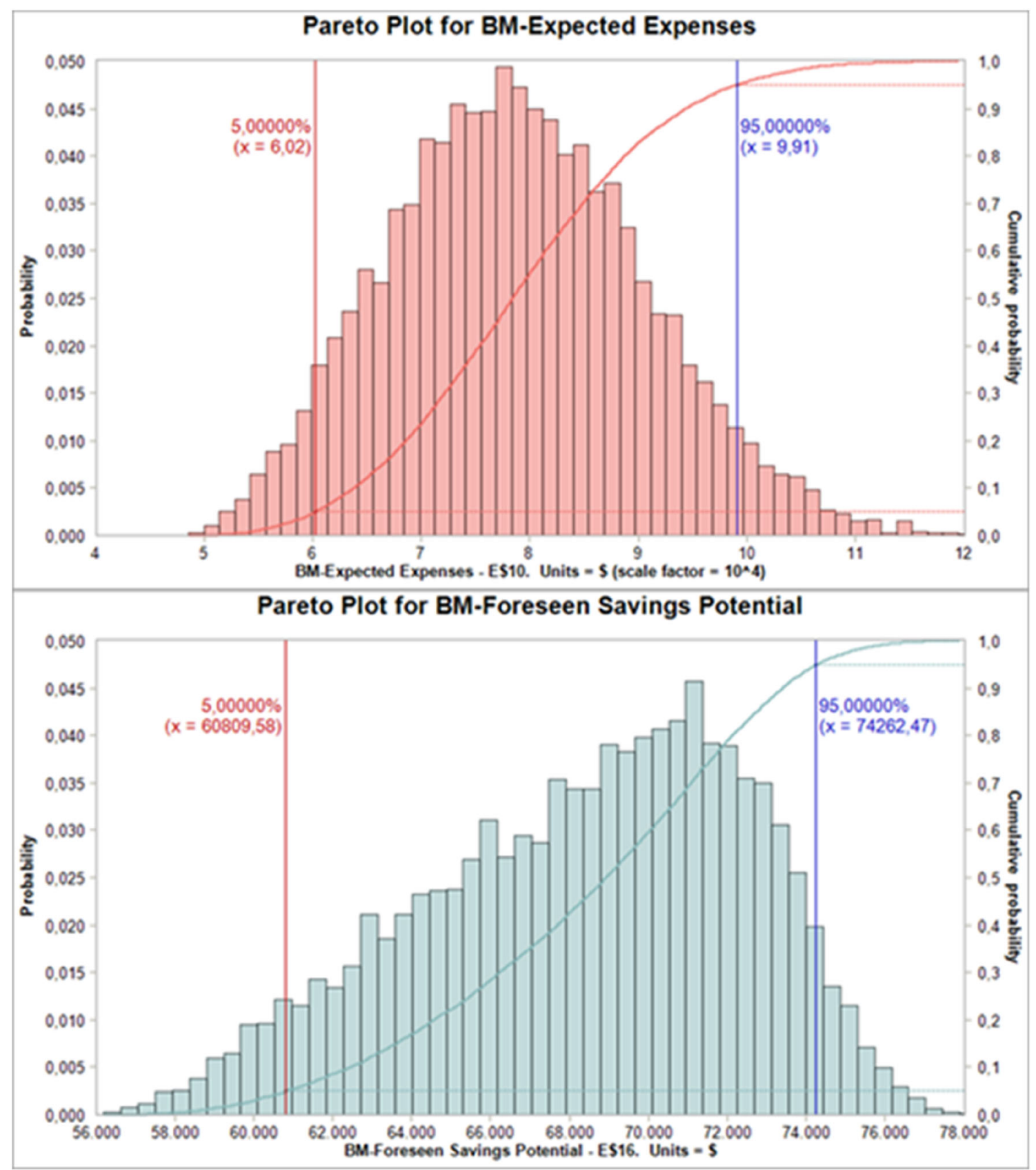

Fig. 10 Pareto Plots developed by ModelRisk for Bit Modifications Solution

curves. The same approach was implemented to develop MI for other solutions.

\section{Constructing Solution Matrix}

Using the described procedure for every individual solution will yield values for the TI and MI listed in Table 1. The solution matrix can be constructed once all the values are known, as shown in Fig. 12.

Based on the results shown in the solution matrix, it is clear that OBM has the highest impact on the bit balling mitigation, thus making it the most appropriate solution from the technical aspect. However, considering its Monetary Indice, it becomes clear that OBM is the least viable solution. Considering a slight difference in technical impact, $\mathrm{AbC}$ arises as a much better solution. On the other extreme, modifying hydraulics may not fail in preventing the Bit Balling at a high success rate as OBM or AbC; nevertheless, it can be considered as a preferable solution in terms of economic impact. Given the assumptions outlined in 4.1 , it is hard to define the most appropriate solution for a particular root cause, mainly as this depends 
Fig. 11 Visual representation of MI for Bit modification solution
MI - Bit modification

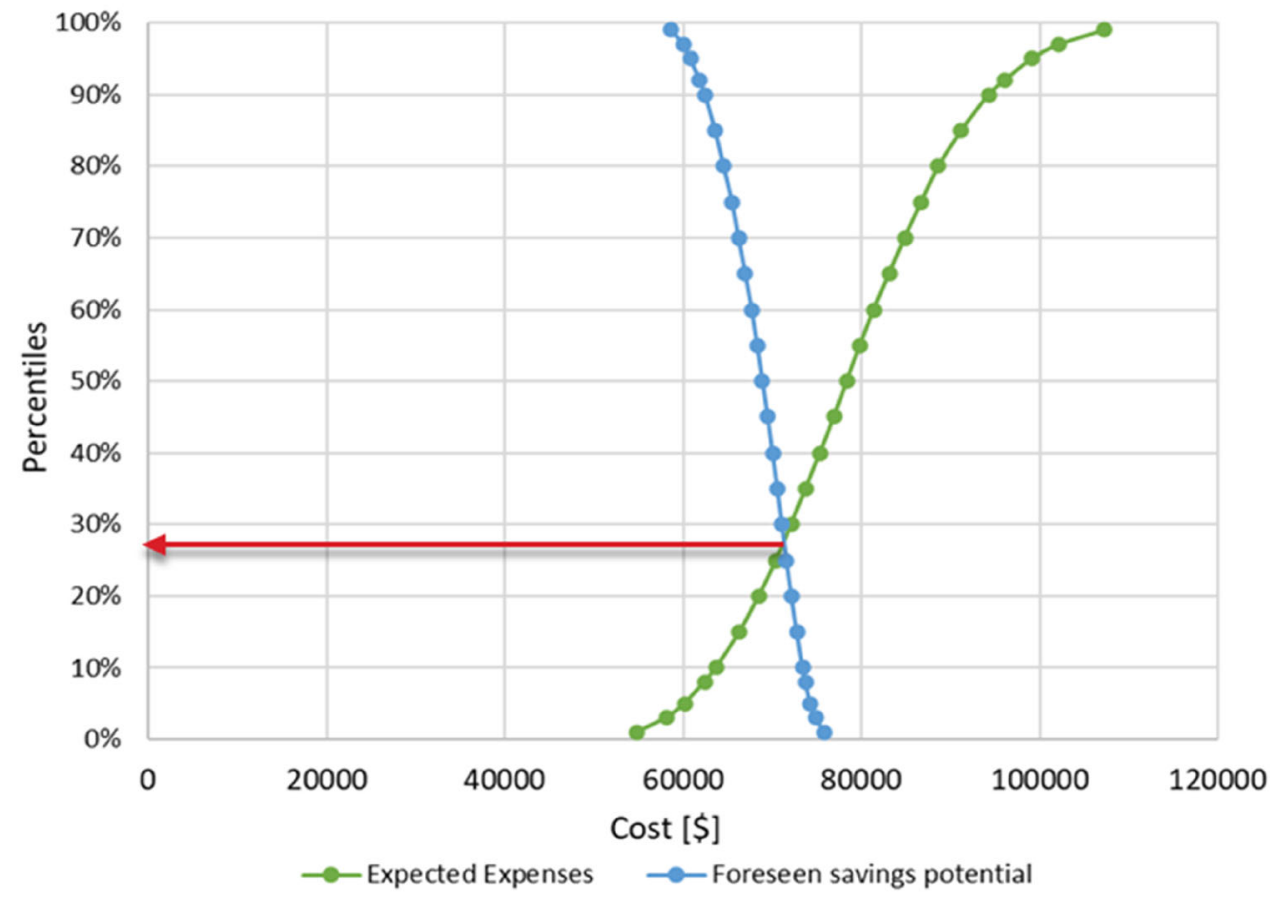

Table 1 Tabular Display of The Technical and Monetary Indices for The Considered Drilling Solutions

\begin{tabular}{lll}
\hline Solution Method & $\begin{array}{l}\text { Technical Indice } \\
(\mathrm{TI})\end{array}$ & $\begin{array}{l}\text { Monetary Indice } \\
(\mathrm{MI})\end{array}$ \\
\hline Bit Modifications (BM) & 0.42 & 0.27 \\
$\begin{array}{l}\text { Hydraulics Optimization (HO) } \\
\text { Improved Water-Based-Mud }\end{array}$ & 0.59 & 0.74 \\
$\quad$ (IWBM) & 0.83 & 0.11 \\
$\begin{array}{l}\text { Oil Based Mud (OBM) } \\
\text { Ant-iballing Coating (AbC) }\end{array}$ & 0.81 & 0 \\
\hline
\end{tabular}

on the well-planning team's preferences. However, it has been seen on the synthetic example that it is possible to vastly enhance the decision-making process while bringing clarity and certainty to the preferred output.

\section{Tool Limitations}

The main limitations of the proposed tool based on the performed case study are summarized in the following points.

1. Indice values are pretty susceptible to input data and should be thoroughly examined for oversight before developing them.

2. Triangular distribution may not correctly represent real-life data distribution, which may be overcome by choosing a different PDF or fitting historical data to yield adequate distribution for calculation purposes.
3. All of the assigned variables, like distribution estimates and Weighted Factors, can easily be biased and thus mask the actual technical performance of proposed solutions. Incorporating more estimates into the PDF model and adopting specific weighted factors for different criteria from the operator's side may help reduce errors in finding the optimum solution for a particular problem.

4. Solutions considered are limited to the user input, i.e., failing to outline all possible solutions for a given problem may leave out the most optimum one.

\section{Conclusion}

During the well-planning phase, the primary purpose of evaluating possible proactive solutions for a given downhole problem is to obtain the most robust and reliable solution among the alternatives. Nevertheless, the selection process is challenging due to the considerable uncertainty associated with the available data at the planning phase, leading to increased risk and non-productive time rather than reducing the overall drilling cost.

This paper introduced a method to rank all possible proactive solutions for a given downhole problem, which can help the drilling engineer to generate an effective plan for combating the downhole issues. The advantages of the presented tool over others are: (1) two key aspects (technical impact, and pay-off probability) are used to assess the 


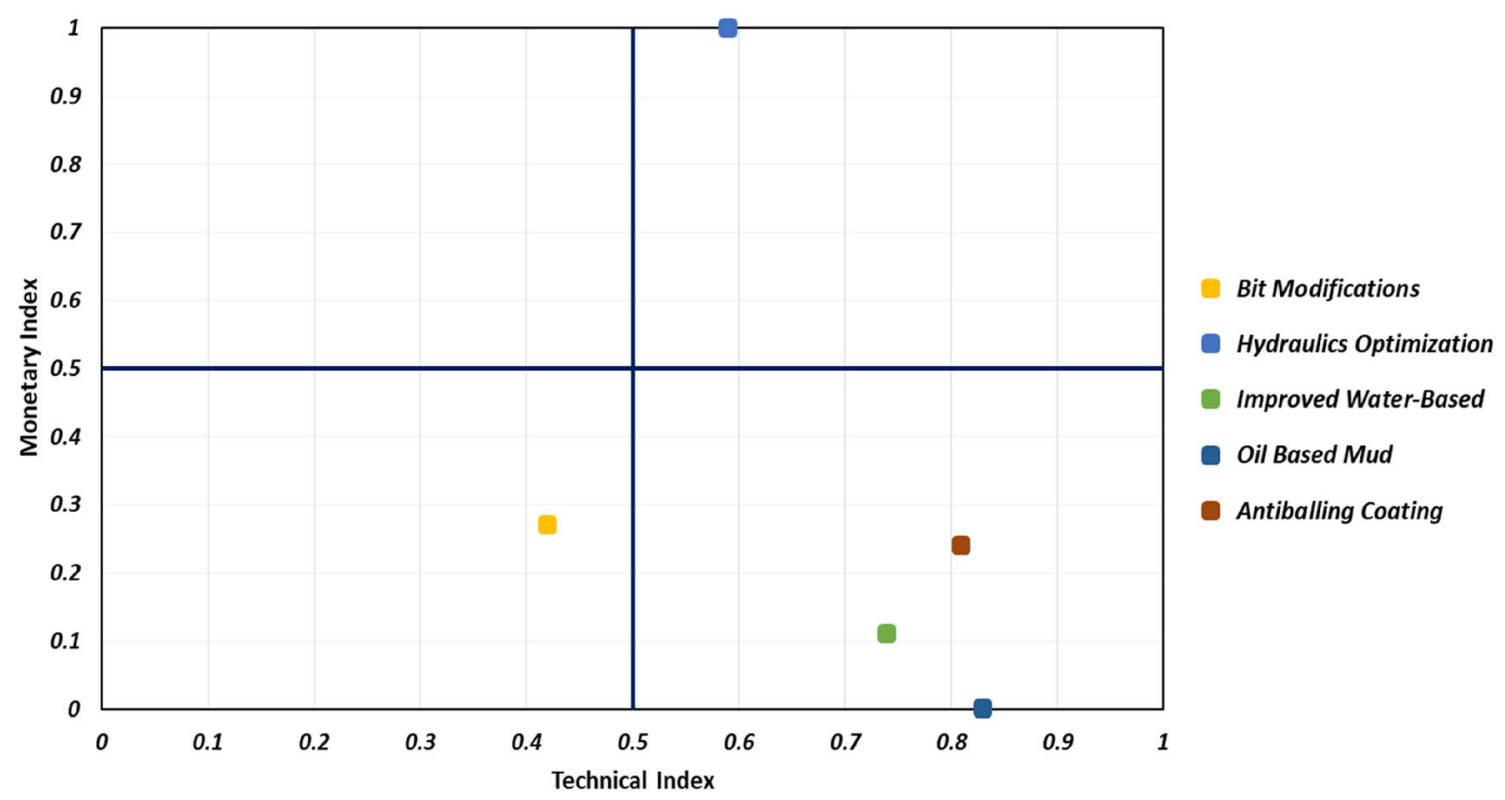

Fig. 12 Integrated Tool Final Solution Matrix output for the Bit Balling

expected performance of the available solutions, (2) uncertainties associated with input data is reduced by utilizing Monte Carlo Simulation in the construction of decision-driving indices, (3) the results are expressed in the form of an evaluation matrix, which comprises a quantitative basis for selecting the optimal drilling solution in terms of both cost and effectiveness among the alternatives, allowing users to easily rank all the considered solutions just through the end-line visualization.

Despite the pointed-out shortcomings, the tool's theoretical applicability was validated through the performed case study, where several solutions were evaluated in their success and viability to mitigate the Bit Balling issue.

\section{Appendix}

Weighted Score formulas

$W S_{1}=\left(\frac{C C_{\min }}{C C}\right) \times C C_{W F}$

$W S_{2}=\left(\frac{C C}{C C_{\max }}\right) \times C C_{W F}$

where:

$W S_{1}$ - Weighted Score for a group of criteria defined with the minimum value.

$W S_{2}$-Weighted Score for a group of criteria defined with the maximum value.

$C C_{W F}$ - Comparative Criteria weighting factor.

$C C_{\text {min }}$ - minimum Comparative Criteria value.

$C C_{\max }$ - maximum Comparative Criteria value.
Funding Open access funding provided by Montanuniversität Leoben.

\section{Declarations}

Conflict of interest On behalf of all authors, the corresponding author states that there is no conflict of interest.

Open Access This article is licensed under a Creative Commons Attribution 4.0 International License, which permits use, sharing, adaptation, distribution and reproduction in any medium or format, as long as you give appropriate credit to the original author(s) and the source, provide a link to the Creative Commons licence, and indicate if changes were made. The images or other third party material in this article are included in the article's Creative Commons licence, unless indicated otherwise in a credit line to the material. If material is not included in the article's Creative Commons licence and your intended use is not permitted by statutory regulation or exceeds the permitted use, you will need to obtain permission directly from the copyright holder. To view a copy of this licence, visit http://creativecommons. org/licenses/by/4.0/.

\section{References}

1. P. L. York, D. M. Prichard, J. K. Dodson, T. Dodson, S. M. Rosenberg, D. Gala, and B. Utama. Eliminating non-productive time associated with drilling through trouble zones. Paper presented at the Offshore Technology Conference, Houston, Texas, (2009) https://doi.org/10.4043/20220-MS

2. S. V. Shokouhi, P. Skalle. Enhancing decision making in critical drilling operations. Paper presented at the SPE Middle East Oil and Gas Show and Conference, Manama, Bahrain, (2009) https://doi.org/10.2118/120290-MS

3. E. Cayeux, B. Daireaux, M. Karimi Balov, S. Haavardstein, L. Magne Stokland, and A. Saasen, Automatic performance analysis and estimation of risk level embedded in drilling operation plans. Paper presented at the SPE Intelligent Energy International 
Conference and Exhibition, Aberdeen, Scotland, UK, (2016) https://doi.org/10.2118/181018-MS

4. S. Thompson, Difference Between a Proactive \& a Reactive Business Strategy. Updated (2019) https://smallbusiness.chron. com/difference-between-proactive-reactive-business-strategy62157.html

5. Prosper Aideyan, Drilling operations: cost and risk management. ISBN 10: 0990683621, Publisher: SigmaQuadrant, January 1, 2015. https://www.abebooks.com/9780990683629/DrillingOperations-Cost-Risk-Management-0990683621/plp

6. C. Dudley, P. Leach, P. Wicker, and S. Anderson, Well planning: a risk management process. Paper presented at the SPE Deepwater Drilling and Completions Conference, Galveston, Texas, USA, (2012) https://doi.org/10.2118/156332-MS

7. A. Aamodt, Knowledge-intensive case-based reasoning in CREEK, in Advances in case-based reasoning ECCBR 2004 Lecture Notes in Computer Science, vol. 3155, ed. by P. Funk, P.A. González Calero (Springer, Berlin, Heidelberg, 2004)

8. A. Sadlier, I. Says, R. Hanson, Automated decision support to enhance while-drilling decision making: where does it fit within drilling automation?. Paper presented at the SPE/IADC Drilling Conference, Amsterdam, The Netherlands, (2013) https://doi.org/ 10.2118/163430-MS

9. M. Giese, R. Bratvold, Probabilistic modeling for decision support in integrated operations. Paper presented at the SPE Intelligent Energy Conference and Exhibition, Utrecht, The Netherlands, (2010) https://doi.org/10.2118/127761-MS

10. T. Matviykiv, V. Teslyuk, Use of influence diagrams for decision support in drilling automation J. Global Res. Computer Sci. 4, 4 (2013) https://www.rroij.com/open-access/use-of-influencediagrams-for-decision-support-in-drilling-automation-1-7.pdf

11. S. Yasseri, (2017) Drilling risk identification filtering ranking and management. Int. J. Coast. Offshore Eng. https://doi.org/10. 18869/acadpub.ijcoe.1.1.17

12. A. Kumaraningrum, A. Indra, D. Putri, A. Mohd, H. Hermansyah, Semi-quantitative risk analysis of a normally unmanned installation facility. J. Petrol Explor. Prod. Technol. 9, 3135-3147 (2019). https://doi.org/10.1007/s13202-019-0711-0

13. W. Jiang, R. Samuel. Minimization of downhole tool failures using nash equilibrium and downhole data. Paper presented at the SPE/IADC Drilling Conference and Exhibition, The Hague, The Netherlands (2017) https://doi.org/10.2118/184687-MS

14. J. Zhao, Y. Shen, W. Chen, Z. Zhang, and S. Johnston, Machine learning-based trigger detection of drilling events based on drilling data. Paper presented at the SPE Eastern Regional Meeting, Lexington, Kentucky, USA, (2017) https://doi.org/ 10.2118/187512-MS

15. P. Filho, F. Tito, J. Guaraci Filardo, Machine learning applied on fishing occurrence prediction. Paper presented at the Offshore Technology Conference Brasil, Rio de Janeiro, Brazil (2019) https://doi.org/10.4043/29700-MS

16. G. Guidry, K. Spezia, and G. Salmon, Collaborative Real-Time analysis to reduce non-productive time. Paper presented at the SPE Annual Technical Conference and Exhibition, Dallas, Texas, USA, (2018) https://doi.org/10.2118/191631-MS

17. R. Hogan. Probability distributions for measurement uncertainty. Updated (2015) https://www.isobudgets.com/probabilitydistributions-for-measurement-uncertainty

18. ModelRisk, Monte Carlo simulation software that makes quantitative risk analysis intuitive, (vosesoftware), https://www.vose software.com/products/modelrisk/

19. K. Bybee, Low-friction coating reduces PDC bit balling. J. Pet. Technol. 54, 35-36 (2002). https://doi.org/10.2118/12020035-JPT

20. S. Lashari, A. Borujeni, E. Fathi, T. Sun, R. Rahmani, M. Khazaeli, Drilling performance monitoring and optimization: a data-driven approach. J. Petrol Explor. Prod. Technol. 9, 2747-2756 (2019). https://doi.org/10.1007/s13202-019-0657-2

Publisher's Note Springer Nature remains neutral with regard to jurisdictional claims in published maps and institutional affiliations. 